\title{
Study on Extenics Information Fusion Method and It's Application
}

\author{
Li Zhong Wang Ailing Qi Furong \\ Institute of Disaster Prevention Science and Technology. Sanhe, Hebei. China \\ Email:lizhong@fzxy.edu.cn
}

\begin{abstract}
This paper makes analysis on theories of D-S evidential reasoning, rough sets, and extenic sets; and indicates that there are some similarities among the three theories in defining uncertainty sets. Therefore, it's feasible to introduce relevant theories and methods of extenics into information fusion and a method of extenics fusion (MEF) is presented as well. The method combines extenic correlation function with Dempster rule and is consider ed a good solution for problems of evidence collision and BPA function in information fusion method based on D-S evidential reasoning. The simulation test shows that MEF is better than the traditional D-S evidential reasoning and is applicable to assess all kinds of problems. Using the method of this paper to evaluate surface water in one area of Norther $n$ China, the results were consistent with the fact.
\end{abstract}

Index Terms- Extenics-fusion; D-S evidential reasoning; $M E F ; B P A$

\section{INTRODUCTION}

In information fusion algorithm based on D-S evidential reasoning, it is often required to compute the basic probability distribution functions (BPA), belief function and plausibility function for all basic evidential bodies. The Dempster rule of combination can put the belief functions of each evidential body together, compute the orthogonal sum of confidence levels from different sources, obtain a new belief function on the target body and form a correct judgment on the objective. It has been difficulty how to choose a proper BPA in the information fusion algorithm based on D-S evidential reasoning, however, whether the BPA is accurate or does not have a direct influence on the fusion result. For this reason, some scholars apply soft methods to obtain BPA and have had some effect [1-3]. Yet, these methods are very complex and not intuitive in calculation. The
Dempster rule requires that the evidential body is independent to each other. Often when conflicts occur, it will be possible for inconsistency between fusion results and intuition. The conflicting evidential bodies need to be revised [4] and the correlation evidential body is converted into independent ones before using Dempster rule. Yet the BPA functions still need to be computed when obtaining the correlation coefficient. Therefore, it is worth to make further study on how to obtain a reasonable BPA intuitively and quickly as well as to resolve the conflicts of evidential bodies.

The Extenics is better used in solving incompatibilities and conflicts [6]. With matter-element theory and correlation function, the extenics usually unites qualitative and quantitative changes together to handle conflicts. This paper introduces the extenics theory into information fusion calculations, defines BPA of D-S evidential reasoning with extenics correlation functions, and combines all BPA with Dempster rule, which makes it simple and feasible to determine the BPA of evidential bodies.

\section{MATHEMATICAL BASIS OF EXTENICS INFORMATION FUSION METHOD}

In a sense, the matter-element theory and information integration are interlinked.

Information fusion is an identification for multiple information sources, uncertain information in the framework of recognition through the focusing element of the combination of different trust. In studying the element theory, you can describe all the features of things as a recognition framework, so that the characteristics of matter-element can be viewed as a proposition, which trust obtained from the information sources. In this sense, the matter-element theory and the information fusion theory has similarities. The matter element emphasizes the characteristics of things, but the information fusion is in favor of obtaining the various 
characteristics. Some basic concepts adopted in the information fusion based on D-S evidential reasoning mainly includes: basic probability assignment function, belief function and plausibility function, among which, the belief function describes the maximum intervals value of supporting evidence and the plausibility function characterizes the minimum intervals value of unsupported evidence, the remaining part between the two values is the believable interval of supporting evidence, $[\mathrm{Bel}(\mathrm{A}), \mathrm{PI}(\mathrm{A})]$, as shown in Fig. 1. It can be seen that the believable interval has been an uncertain set in the D-S evidential reasoning and the fusion computing is in the belief interval.

In the rough set theory, two identified concepts of upper and lower approximation can be used to describe the fuzzy of uncertainty sets. The difference between the two sets is regarded as the uncertainty set, i.e, the boundary field. According to rough set theory, the lower approximation set is called positive field, the complement set of upper approximation is called negative field; the difference between the two sets is called the boundary field. The relationship of the three fields are shown in Fig. 2.

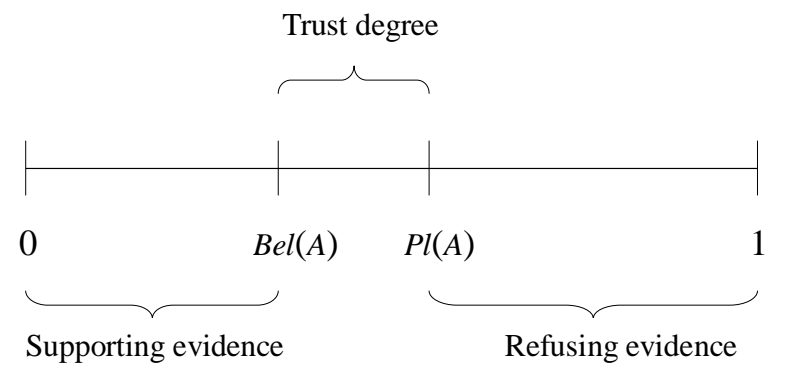

Fig.1 Believable interval of D-S evidence theory

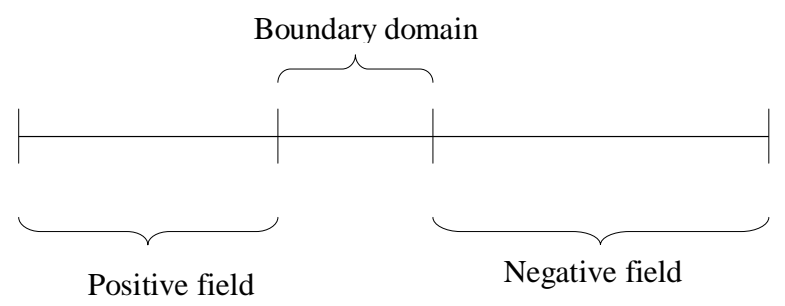

Fig.2 Positive field, negative field and boundary field of rough set theory

Teacher Research Fund of China Seismological Bureau (No.20090105) and Science and Research Programs of Hebei Education Department of China (No. Z2009407)

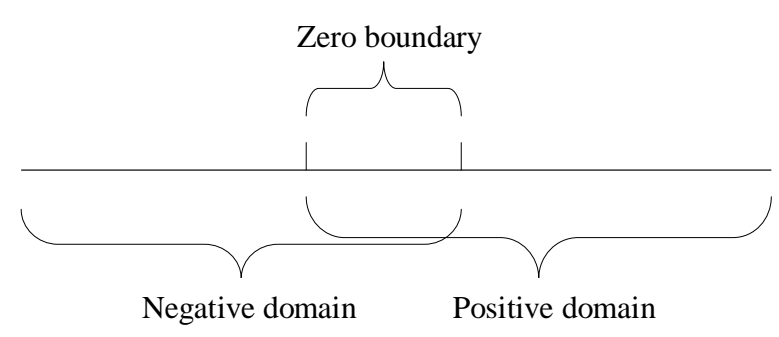

Fig.3 Positive field, negative field and zero field of extenic set theory

The Extenics set can be divided into three parts: positive field, negative field and a zero boundary. The positive field represents matter-element sets that meets requirements, while the negative field refers to the sets that does not meet requirements. The zero boundary means intersection of positive and negative fields, which refers to a specious concept and the state can be changed to satisfied from unsatisfied after a certain transformations as shown in Fig. 3.

From the above description, it is easily seen that there are lots of similarities for the three kinds of sets in characterizing uncertain sets. The D-S evidence theory uses confidence interval to show uncertainty sets and the rough set theory names the uncertainty interval as a boundary field, while the extenics set theory refers the zero boundary as the uncertainty interval. Since both interval of $(-\infty,+\infty)$ and $(0,1)$ have isomorphism relation, the definition of the three are accordant. As we all know that the D-S evidence theory and rough set theory have been widely used in information fusion, it is feasible to introduce the extenics theory into information fusion processing with some matured research methods.

\section{INFORMATION FUSION METHOD OF EXTENICS-BASED D-S EVIDENTIAL THEORY}

Factors affecting change of things usually cover many aspects and the common information accumulation from every single aspect causes qualitative change of things. So we need to make composite consideration for all information of every channel. Shafer said [7]: "The influence on one proposition of evidence should include at least two aspects of information: how the evidence effectively proves the proposition and how the evidence effectively proves its anti-proposition". In the D-S evidential theory, it is difficult to determine the confidence level of one proposition, and usually 
possesses subjectivity. The probability of digitalization cannot be independent of the objective attributes of human judgments, which not exist in our brains before thinking. However, we can construct a digitalized probability with basis of objective evidences as well as make analysis over the evidences during the whole process. Perhaps the evidences are ambiguous in the very beginning and the judgment about propositions is also non-quantifiable, even without any structure and logic, yet, with advancement of understanding and appearance of other evidences, we may identify the reliability level for a related digitalized proposition [5]. While in the extenics, the correlation function can easily characterize the correlation degree between evidence and proposition. So we can introduce the correlation function of extenics theory into the D-S evidential theory to indicate the supporting degree of evidence to a proposition through the degree of correlation between the character value and classical domain or joint domain of matter element.

The analysis shows that a link can be established between extenics correlation function and belief function in D-S evidential theory. So, a simple method can be found to determine the confidence degree when defining the supporting degree of evidence to proposition with their correlation degree to each other, which is also a practical solution in addressing the basic probability assignment in D-S theory. The algorithm of introducing extenics theory to the information fusion is called method of extenics fusion (MEF).

Based on the above analysis, algorithm is designed as follows:

\section{Step1. Setting matter-element model}

According to the definition of matter-element, it is limitless to describe the characteristics of a thing, but to describe a thing with only finite characteristics. Supposing that there are $\mathrm{n}$ features of a thing, denoted as $\left\{C_{1}, C_{2}, \ldots, C_{n}\right\}$. So the element-model is set up as follows:

$$
R=\left[\begin{array}{cc}
N & C_{1} \\
& C_{2} \\
& \cdots \\
& C_{n}
\end{array}\right]
$$

Step2. Determining the classical domain of matterelement

According to the matter-element theory, the scope of typical value for a thing in keeping its own characteristic is called to be classical domain of matter element. The classical domain can be divided into multiple subfields under its different characteristics of matter element. Each subfield is called level or rank for the purpose of detailed analyzing matters. The classical domain of matter-element is generally an interval consisted by a pair of data. For the non-digital text, digital processing needs to be done to calculate facilitate. Let's suppose the matter-element classical domain with $n$-feature $\left\{C_{1}\right.$, $\left.\mathrm{C}_{2}, \ldots, \mathrm{C}_{\mathrm{n}}\right\}$ is as follows:

$$
R_{0}=\left[\begin{array}{ccc}
N & C_{1} & <a_{1}, b_{1}> \\
& C_{2} & <a_{2}, b_{2}> \\
\cdots & \ldots \\
C_{n} & <a_{n}, b_{n}>
\end{array}\right]
$$

A classification is usually done in evaluating problems. Supposing the target is divided into $\mathrm{m}$ levels with $\left\{\mathrm{I}_{1}\right.$, $\left.I_{2}, \ldots, I_{m}\right\}$ of the corresponding classical domain, which can be presented with matrix as follows:

$$
R_{0}=\left[\begin{array}{ccccc}
N & I_{1} & I_{2} & \ldots & I_{m} \\
C_{1}<a_{11}, b_{11}><a_{12}, b_{12}>\ldots<a_{1 m}, b_{1 m}> \\
C_{2}<a_{21}, b_{21}><a_{22}, b_{22}>\ldots<a_{2 m}, b_{2 m}> \\
\ldots & \ldots & & \\
C_{n}<a_{n 1}, b_{n 1}><a_{n 2}, b_{n 2}>\ldots<a_{n m}, b_{n m}>
\end{array}\right]
$$

Step3. Determining the joint domain of matterelement

The joint domain of matter-element is the scope of the quantitative change which keeps its quality. When the variation of quantity is within the scope of joint domain, the quality will maintain its stability, once beyond the joint domain, a qualitative change will happen. So, the joint domain is the union of all classical domains. If expressed with above matter-element, then the joint domain will be:

$$
R_{p}=\left[\begin{array}{ccc}
N & C_{1} & <c_{1}, d_{1}> \\
& C_{2} & <c_{2}, d_{2}> \\
\cdots & \ldots \\
& C_{n} & <c_{n}, d_{n}>
\end{array}\right]
$$


Clearly, according to the level classical domains, there is $c_{i}=a_{i 1}, d_{i}=b_{i m}, i=1,2, \ldots, n$.

Step4. Determining the matter-element to be evaluated

The characteristics of matter-element can be obtained through inspection, measure, test, experience, analysis, computation and other ways. Assuming the matter-element to be evaluated is:

$$
R=\left[\begin{array}{ccc}
N, & C_{1} & v_{1} \\
& C_{2} & v_{2} \\
\ldots & \ldots \\
& C_{n} & v_{n}
\end{array}\right]
$$

Step5. Computing the correlation degree of matterelement for classical domains

Assuming that the classical domain of feature $\mathrm{C}$ for matter-element $\mathrm{N}$ is $\langle\mathrm{a}, \mathrm{b}\rangle$, the joint domain is $\langle\mathrm{c}, \mathrm{d}\rangle$, the corresponding value to be evaluated is $\mathrm{v}$, and so the correlation degree can be computed as follows:

$$
K(v)=\left\{\begin{array}{cc}
-2 \times \rho(v,<a, b>) /(b-a) & v \in<a, b> \\
\frac{\rho(v,<a, b>)}{\rho(v,<c, d>)-\rho(v,<a, b>)} & v \notin<a, b>
\end{array}\right.
$$

Here, $\rho(v,<a, b>)=|v-(a+b) / 2|-(b-a) / 2$

$$
\rho(v,\langle c, d>)=|v-(c+d) / 2|-(d-c) / 2
$$

For evaluation, the above correlation value will form a matrix of $n \times m$ that the column represents feature, while the row means correlation degree. That is:

$$
K=\stackrel{C_{2}}{C_{1}} \underset{C_{n}}{C_{n}}\left[\begin{array}{cccc}
K_{11} & K_{12} & \ldots & K_{1 m} \\
K_{21} & K_{22} & \ldots & K_{2 m} \\
\ldots & \ldots & \ldots & \ldots \\
K_{n 1} & K_{n 2} & \ldots & K_{n m}
\end{array}\right]
$$

Step6. Calculating the belief degree of evidential body

An evidential body consists $(A, m(A))$, where $A$ is proposition and $m(A)$ is the reliability distribution function supporting $A$. With the correlation function of classical domain for the matter-element, we can obtain the belief degree of a proposition, i.e; one feature value belongs to a specific classical domain. Supposing k is the correlation degree between a feature value $\mathrm{v}$ and one classical domain, there is $-\bullet<k<+\bullet$ from the definition of correlation function of (5). Using S-function to transform and making normalization with row:

$$
L=\left[\begin{array}{cccc}
L_{11} & L_{12} & \ldots & L_{1 m} \\
L_{21} & L_{22} & \ldots & L_{2 m} \\
\ldots & \ldots & \ldots & \ldots \\
L_{n 1} & L_{n 2} & \ldots & L_{n m}
\end{array}\right]
$$

Thus, in (7), the element in each row is the distribution of confident degree for feature value in supporting classification proposition.

Step7. Making information fusion using Dempster rule

According to Dempster rule [7], the confident degree of different evidential bodies shall be combined a whole distribution of credibility level, which produces a new evidential body. Thus the final recognition result is generated as well.

The flow chart of this algorithm is shown in Fig.2.

IV. EXPERIMENTAL SIMULATION AND ANALYSIS OF MEF ALGORITHM

Experimental simulation is needed to verify the validity of the extenics fusion as well as an analysis over the data and making comparison with the result of other method.

\section{A. Experimental simulation of MEF algorithm}

To achieve MEF, we developed a simulation program using Matlab and made verification as an example with data provided by literature [8].

Tab.1 shows the surface water indexes of one river during the first three months in 2002. Each test item was a group of evidence in water quality checking. The surface water is divided into five categories according to national standards [9]. Due to the bigger of dissolved oxygen the better among all testing items, we used its countdown for processing data.

I.J.M odern Education and Computer Science, 2011, 2, 29-36 


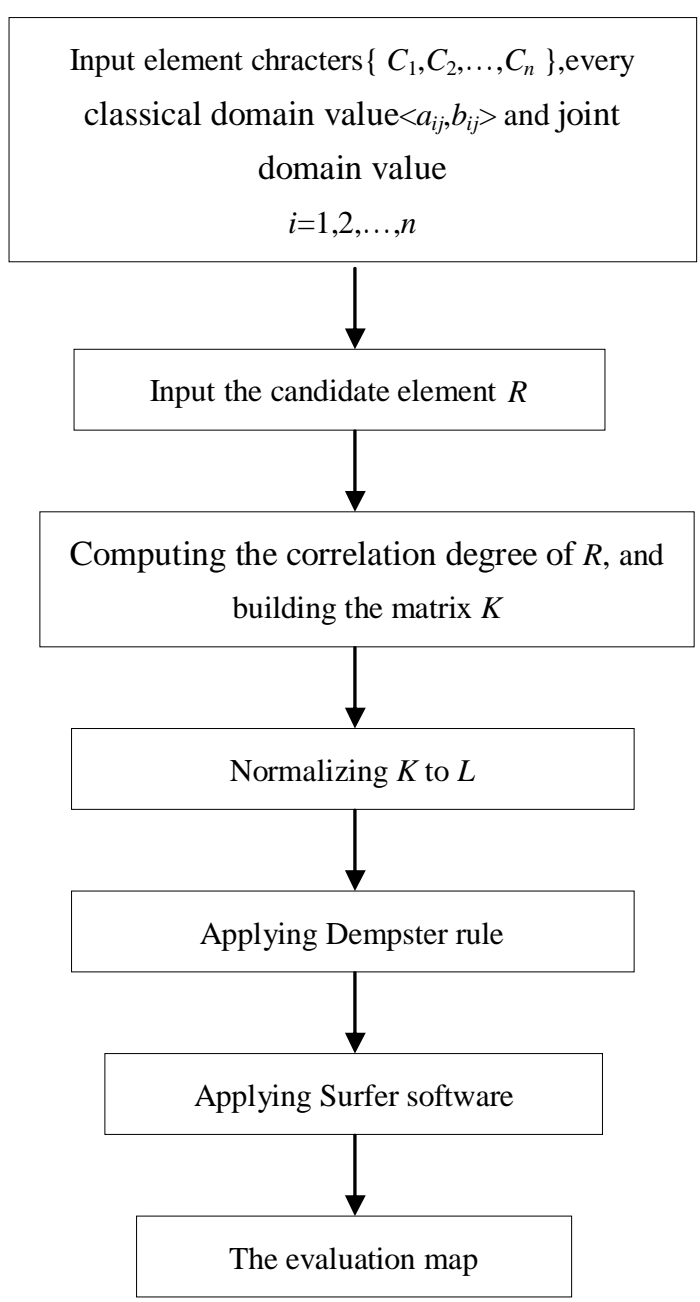

Fig.2 Algorithm flow chart of extenics fusion

Tab.1 Water quality index of a river $\quad(\mathrm{mg} / \mathrm{L})$

\begin{tabular}{|c|c|c|c|c|}
\hline & BOD & Per manganate & $\begin{array}{c}\text { Dissolved } \\
\text { xygen }\end{array}$ & A mmonia \\
\hline J an. & 0.95 & 2.28 & 10.8 & 0.213 \\
\hline Feb. & 1.58 & 2.55 & 10.55 & 0.387 \\
\hline M ar. & 0.9 & 3.03 & 10.5 & 0.37 \\
\hline
\end{tabular}

In the information fusion of D-S evidential theory, we need to know the basic belief level of each sample with regard to the surface water level and make fusion calculation using data of literature [8]. For purpose of comparison, we conducted a water quality evaluation with 3 methods (D-S evidential theory, extenics evaluation and MEF). The results are shown in Tab. 2.
Tab..2 Surface water assessment results for a river from

\begin{tabular}{|l|c|c|c|}
\hline & Jan.to Mar. & Feb. & M ar. \\
\hline $\begin{array}{l}\text { D-S evidential } \\
\text { theory }\end{array}$ & I & I & I \\
\hline $\begin{array}{l}\text { Extenics } \\
\text { evaluation }\end{array}$ & I & II & II \\
\hline MEF & I & II & II \\
\hline
\end{tabular}

As can be seen in Tab.2, the results of extenics evaluating method and extenics fusion are consistent, while result of D-S evidential theory is different.

The analysis shows D-S evidential theory requires a basic reliability function value of all evidence bodies as $\mathrm{s}$ base, while the values are usually given artificially, which consequentially leads to the inaccuracy of judgment.

\section{B. Algorithm analysis}

Firstly, misjudgment will happen often due to the improper handling to the conflicting evidence of D-S evidence theory. In the multi-source information fusion, since the manifold information delivers diversity and uncertainty of information, this is the root cause of why we use information fusion. However, the diversity and uncertainty of information brings more conflicting possibilities to the source evidence. This kind of conflict is not caused by a single focal element, it could be the errors of two different evidences, or some unknown reason or uncertainties, or some external disturbances. According to Dempster rule, there is no processing when evidence conflicting happened, which would produce inconsistent conclusion compared with intuition. In fact, the debate on the Dempster rule has always existed in the field of information fusion and currently there are two main options. One option believes the Dempster rule is improper in dealing with two intersectional BPA functions of focal elements under a condition of empty set and results misjudgment. The solution to this problem is to redistribute the conflicted evidence [4]. The other option believes that the Dempster rule is not wrong. Yet, a preprocessing shall be done to the evidence when conflicts happened in order to prevent conflicts from occurrence [7]. The extenics-based information fusion 
method of this paper belongs to the latter option.

The second is robust performance. When small changes occur in giving basic probability assignment value to a focal element, the results of Dempster rule will change sharply and show weak stability, which reflect that the Dempster rule is extremely sensitive to the values of basic belief function.

Lastly, the computing complexity of the algorithm. The MEF consists two parts in calculation: confidence distribution of category proposition and Dempster combining calculation. The main work in the first part is to obtain consumption of correlation matrix with a computation of $m n+m n+m n=0(m n)$. In the second part, the computation of information fusion for $n$ sensors is $O\left(n^{2}\right)$, taking into account $m$, the evaluation category and $\mathrm{O}\left(\mathrm{mn}^{2}\right)$, parts of the computation amount of information fusion. So the total computation is $O(m n)+O\left(m n^{2}\right)=$ $\mathrm{O}\left(\mathrm{mn}^{2}\right)$.

\section{AN APPLICATION EXAMPLE}

In 2007, we investigated the surface water in an area in the north of China, and got 19 samples data in the table 3 .

According to the National Standard (GB3838-2002), the surface water is divided into five categories in China, as shown in table 4.

Tab.3 Data of surface water

Unit: $\mathrm{mg} / \mathrm{L}$

\begin{tabular}{|c|c|c|c|c|}
\hline No. & $\begin{array}{c}\text { Ammoni } \\
\mathrm{a}\end{array}$ & $\begin{array}{c}\text { Permanganat } \\
\mathrm{e}\end{array}$ & $\begin{array}{c}\text { Dissolve } \\
\text { d oxygen }\end{array}$ & BOD \\
\hline 1 & 2.4 & 0.05 & 6.95 & 23.2 \\
\hline 2 & 0.02 & 0.06 & 7.19 & 3.2 \\
\hline 3 & 0.01 & 0.07 & 7.28 & 5.36 \\
\hline 4 & 0.04 & 0.17 & 7.19 & 6.24 \\
\hline 5 & 0.24 & 0.06 & 7.1 & 6.7 \\
\hline 6 & 0.16 & 0.04 & 7.16 & 5.6 \\
\hline 7 & 0.04 & 0.06 & 7.15 & 5.04 \\
\hline 8 & 0.6 & 0.14 & 7.16 & 33.2 \\
\hline 9 & 15 & 1.84 & 6.82 & 23.67 \\
\hline 10 & 0.12 & 0.05 & 6.84 & 7.17 \\
\hline 11 & 0.001 & 0.01 & 6.8 & 2.42 \\
\hline 12 & 0.001 & 0.01 & 6.78 & 2.42 \\
\hline 13 & 0.001 & 0.02 & 6.82 & 4.08 \\
\hline 14 & 0.001 & 0.01 & 6.84 & 2.25 \\
\hline 15 & 0.001 & 0.03 & 6.81 & 2.17 \\
\hline
\end{tabular}

\begin{tabular}{|c|c|c|c|c|}
\hline 16 & 0.001 & 0.01 & 6.96 & 1.83 \\
\hline 17 & 7.5 & 1.01 & 6.95 & 4.33 \\
\hline 18 & 6 & 0.06 & 6.99 & 2.92 \\
\hline 19 & 11 & 2.92 & 7.01 & 5.34 \\
\hline
\end{tabular}

Using of the extension fusion algorithm proposed in this paper to evaluate the surface water in the region, we can get the water quality classification of every sample, as shown in table 5 .

Tab.4 The standard content of several substances

\begin{tabular}{|c|c|c|c|c|c|}
\hline & I & II & III & IV & V \\
\hline Ammonia & $\unlhd 0.15$ & $\unlhd 0.5$ & $\leq$ & $\unlhd .5$ & $\underline{2}$ \\
\hline Permanganate & $\underline{s}$ & $\leq 4$ & 56 & $\leq 10$ & $\leq 15$ \\
\hline Dissolved oxygen & $\geq 7.5$ & 26 & 25 & $\geq 3$ & $\geq 2$ \\
\hline BOD & $\leq 3$ & $\leq 3$ & $\leq 4$ & 56 & $\leq 10$ \\
\hline
\end{tabular}

Tab.5 Classification of surface water quality with MEF

\begin{tabular}{|c|c|c|c|}
\hline No. & Class & No. & Class \\
\hline 1 & III & 11 & I \\
\hline 2 & I & 12 & I \\
\hline 3 & I & 13 & I \\
\hline 4 & I & 14 & I \\
\hline 5 & II & 15 & I \\
\hline 6 & I & 16 & I \\
\hline 7 & I & 17 & II \\
\hline 8 & II & 18 & I \\
\hline 9 & III & 19 & I \\
\hline 10 & I & & \\
\hline
\end{tabular}

Applying Surfer software to process the classified data, we can obtain the evaluation results of surface water in the area, shown in figure 3 . This result is very consistent with the actual situation. 


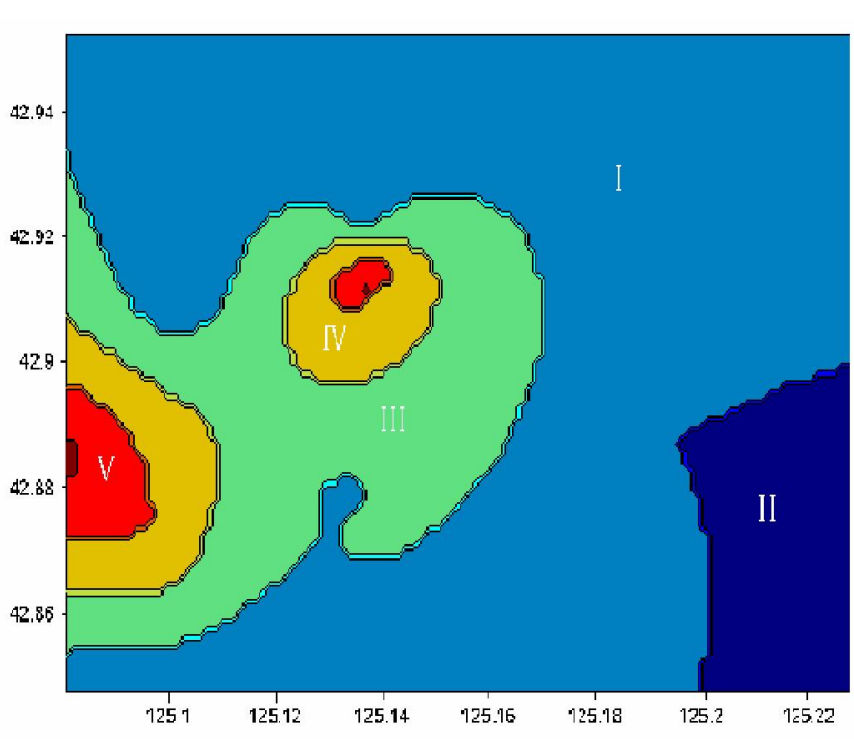

Fig.3 The evaluation map of surface water

\section{CONCLUSION}

It is similar that three methods of rough set, D-S evidence theory and extenics describe uncertain sets. So, it has been feasible to introduce the extenics theory into information fusion. The extenics fusion can combine the matter-element analysis theory, principles of the extenics correlation functions and the Dempster rule of combination, which can solve problems of evidence conflicts of information fusion based on D-S evidential theory and obtain of BPA functions. It can also reduce the influence of human intervention. The simulation test shows that MEF is better than the traditional D-S evidential reasoning. The calculation method is simple and intuitive and is suitable for solving various kinds of evaluations.

\section{REFERENCES}

[1] Wu Xiaoping, Ye Qing, Liu Lingyan. "Dempster-Shafer Theory of Evidence Based on Improved BP and Its Application". Journal of Wuhan University of Technology. vol. 29, PP. 158-161, May 2007.(in Chinese)

[2] Zhong Lu-hong, Wu Wen, Li Xingguo,et al. "Research on Fuzzy Infomation Fusion of Multi-sensor Detecting Systems". Journa 1 of Detection \& Control. vol.2, PP. 42-45, February

\section{3. (in Chinese)}

[3] Salzenstein F, Boudraa A O. "Unsupervised multisensor data fusion approach[C], International Symposium on Signal Processing and its Applications", Kuala Lumpur, Malaysia, pp.152-154. August 2001.

[4] Zhang Qi, Gu Weikang Liu Jilin. "Vision Informayion Fusion Based on Dempster-Shafer Evidence Inference Theory in ALV". Chinese J Computers. vol. 22. pp. 193-198. February 1999 (in Chinese)

[5] Sun Quan, Ye Xiuqing, Gu Weikang. "A New Combination Rule s of Evidence Theory". ACTA ELECTRONICA SINICA. Vol. 28. pp. 117-120. August 2000

[6] Cai Wen, Yang Chunyan, Lin Weichu. "Extenics Engeering Methods". Bei Jing:Science Press. 2000. (in Chinese)

[7] Lawrence A. Klein. "Theory on Fuzzy Information Fusion of Multi- sensor and Its Application". Bei Jing: Beijing Institute of Technology Press.2004 (in Chinese)

[8] Lin Z G, Xu L Z, Huang F C, et al. "Multi-Source Monitoring Data Fusion and Assessment Model on Water Environment". Proceedings of the $3^{\text {rd }}$ International Conference on Machine Learning and Cyber- netics, Shanghai, pp. 26-29. August 2004

[9] The People's Republic of China National Standards: Surface Water Quality Standards(GB3838-2002) (in Chinese)

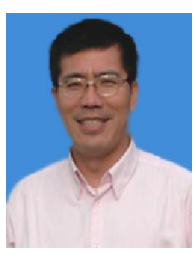

Professor Li Zhong was born in Zhu-cheng city, Shan dong province of China. He obtained his Ph.D. degree in Earth Information Science from China University of Mine and Technology (Beijing) at 2007. His first degree is Bachelor of Science in Basic Mathematics from Shandong University at 1987 and master degree is Master of Industry in Mechanics from Dalian University of Technology at 1990.

He works in Dean of Information Engineering and Computer Science, Institute of Disaster Prevention of China, from 2008. Prior to joining Institute of Disaster Prevention of China, Prof. Li is a associate professor in 
Weifang University, Engineer in Huaguang Company. In 2002, he was a visiting scholar in the e-government research in State Information Center of China. His specialties include, but not limited to, data mining, Artificial Intelligence, $3 \mathrm{~S}$ technology.

Prof. Li Zhong is a fellow of China Computer Federation and a member of Intelligent Computing Council of Operations Research Society of China.

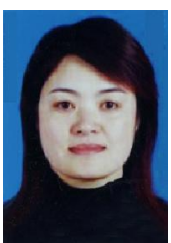

Lecturer Wang Ailing was born in Zhu-cheng city, Shan dong province of China. She obtained her degree in English Literature from Yantai University at 1992.

She always works in Institute of Disaster Prevention of China from 1992. Her specialty is English Translation.

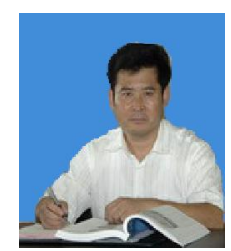

Professor Qi Furong was born in Zaoqiang city, Hebei province of China. He obtained his Ph.D. degree in Geological Engineering from China University of Mine and Technology(Beijing) at 2010. His first degree is Bachelor of Science in Hydrogeology and Engineering Geology from East China Institute of Geolog.

He always works in Institute of Disaster Prevention of China from 1983. His specialties include, but not limited to, hydrogeology, information engineering, etc.

Prof. Qi Furong is a member of Undergraduate Assessment team of Chinese Ministry of Education 